\title{
Bim regulation may determine hippocampal vulnerability after injurious seizures and in temporal lobe epilepsy
}

\author{
Sachiko Shinoda, ${ }^{1,2}$ Clara K. Schindler, ${ }^{1}$ Robert Meller, ${ }^{1}$ Norman K. So, ${ }^{3}$ Tomohiro Araki, ${ }^{1,2}$ \\ Akitaka Yamamoto, ${ }^{1,2}$ Jing-Quan Lan, ${ }^{1}$ Waro Taki, ${ }^{2}$ Roger P. Simon, ${ }^{1}$ and David C. Henshall ${ }^{1}$ \\ ${ }^{1}$ Robert S. Dow Neurobiology Laboratories, Legacy Research, Portland, Oregon, USA. 2Department of Neurosurgery, Mie University School of Medicine, \\ Tsu, Mie, Japan. ${ }^{3}$ Oregon Comprehensive Epilepsy Program, Neurological Sciences Center, Portland, Oregon, USA.
}

\begin{abstract}
Programmed cell death pathways have been implicated in the mechanism by which neurons die following brief and prolonged seizures, but the significance of proapoptotic Bcl-2 family proteins in the process remains poorly defined. Expression of the death agonist Bcl-2-interacting mediator of cell death (Bim) is under the control of the forkhead in rhabdomyosarcoma (FKHR) transcription factors. This prompted us to examine the response of this pathway to experimental seizures and in hippocampi from patients with intractable temporal lobe epilepsy. A short period of status epilepticus in rats that damaged the hippocampus activated FKHR/FKHRL-1 and induced a significant increase in expression of Bim. Blocking of FKHR/FKHRL-1 dephosphorylation after seizures improved hippocampal neuronal survival in vivo, and Bim antisense oligonucleotides were neuroprotective against seizures in vitro. Inhibition of Akt increased the FKHR/Bim response and DNA fragmentation within the normally resistant cortex. Analysis of hippocampi from patients with intractable epilepsy revealed that Bim levels were significantly lower than in controls and FKHR was inhibited; we were able to reproduce these results experimentally in rats by evoking multiple brief, noninjurious electroshock seizures. We conclude that Bim expression may be a critical determinant of whether seizures damage the brain, and that its control may be neuroprotective in status epilepticus and epilepsy.
\end{abstract}

\section{Introduction}

Prolonged seizures (status epilepticus) and the brief and/or repetitive seizures associated with epilepsy are known to cause neuronal loss in the brain (1). This process may underlie the progressive reductions in hippocampal volume seen in some neuroimaging studies of epilepsy patients with frequent or intractable seizures (2). Insight from experimental modeling suggests that such neuronal loss may cause cognitive dysfunction (3), a problem common to patients with poorly managed seizures (4), and worsen seizure severity in epilepsy (5). Significantly, neuronal loss can be directly related to the emergence of spontaneous recurrent seizures in epilepsy (6).

Programmed cell death pathways may be responsible for a significant component of seizure-induced neuronal loss (1, 7). Data from experimental seizure models and now human studies reveal the activation of several effectors of apoptosis in the brain (8-11). Further, molecular or pharmacological intervention in these pathways can reduce seizure-induced neuronal loss (8-11) and may modify the emergent epilepsy phenotype after seizures $(12,13)$.

$\mathrm{Bcl}-2$ family proteins regulate the initiation and progression of programmed cell death (14). Bcl-2-interacting mediator of cell death (Bim) (15), also known as Bod (16), is a potent proapoptotic

Nonstandard abbreviations used: B cell lymphoma (Bcl); Bcl-2-associated death promoter (Bad); Bcl-2-associated X protein (Bax); Bcl-2-homologous antagonist/ killer (Bak); Bcl-2 homology domain 3 (BH3); Bcl-2-interacting mediator of cell death (Bim); BH3-interacting domain death agonist (Bid); cornu ammonis (CA); electroencephalogram (EEG); forkhead in rhabdomyosarcoma (FKHR); kainic acid (KA); 27-kDa Cdk-inhibitory protein 1 (p27 $\left.{ }^{\mathrm{Kip} 1}\right)$; lactate dehydrogenase (LDH); maximal electroshock seizure (MES); sodium orthovanadate (SOV).

Conflict of interest: The authors have declared that no conflict of interest exists.

Citation for this article: J. Clin. Invest. 113:1059-1068 (2004).

doi:10.1172/JCI200419971. member of the Bcl-2 homology domain 3 (BH3) subfamily that can promote neuronal programmed cell death $(17,18)$. Bim is unusual in having numerous splice variants and unique functions that are not compensated for by other BH3-only proteins $(15,19)$. Bim resides sequestered to the microtubule-associated dynein motor complex (20) and, when released, promotes Bax- and caspasedependent apoptosis, largely via neutralizing antiapoptotic Bcl-2 family proteins $(15,16,18,19)$.

Bim expression levels are regulated by transcription factors of the forkhead in rhabdomyosarcoma (FKHR) family that include FKHR (FOXO1) and FKHRL-1 (FOXO3a) (21, 22). In turn, forkhead protein functions are negatively regulated via phosphorylation by $\operatorname{Akt}(23,24)$. Both Bim and the forkhead transcription factors are now implicated in the regulation of neuronal death (18, $22,25)$. Since the molecular determinants of neuronal loss following seizures have significant implications for how we treat status epilepticus, brain injuries known to provoke epilepsy, and perhaps epilepsy itself, we examined the forkhead/Bim pathway in experimental seizure models and hippocampal resections from patients with intractable temporal lobe epilepsy.

\section{Methods}

Seizure model in vivo. All animal procedures were performed in a facility accredited by the Association for Assessment and Accreditation of Laboratory Animal Care in accordance with protocols approved by the Institutional Animal Care and Use Committee and the principles outlined in the NIH Guide for the Care and Use of Laboratory Animals. Studies were performed as previously described (8) with some modifications. Seizures were focally evoked in adult male Sprague-Dawley rats (280-350 g) by unilateral stereotaxic microinjection of kainic acid (KA; Sigma-Aldrich, St. Louis, Mis- 
souri, USA) into the basolateral amygdala nucleus. Following anesthesia, intubation, and vein catheterization, animals were placed in a stereotaxic frame. Two electrodes (Plastics One Inc., Roanoke, Virginia, USA) were then affixed to the skull bitemporally over the hippocampi and a third was placed across the frontal cortex for electroencephalogram (EEG) recordings (Grass model 8-16; Astro-Med Inc., West Warwick, Rhode Island, USA). A craniectomy was also performed for placement of the injection cannula. The animal was then removed from the frame, and anesthesia was discontinued. EEG recordings were commenced, and then a 31-gauge internal cannula (Plastics One Inc.) was inserted into the lumen of the guide to inject $\mathrm{KA}(0.1 \mu \mathrm{g}$ in $0.5 \mu \mathrm{l}$ saline vehicle $)$ into the amygdala. Nonseizure control animals underwent the same surgical procedure but received intra-amygdala vehicle injection. The EEG was continuously monitored until diazepam $(30 \mathrm{mg} / \mathrm{kg}$ i.v. $)$ was administered to terminate seizures after 40 minutes. The EEG was further monitored for up to 1 hour to ensure seizure cessation.

Sodium orthovanadate and LY294002 studies. In vivo inhibition of FKHR/FKHRL-1 dephosphorylation was accomplished by icv infusion of the phosphatase inhibitor sodium orthovanadate (SOV; Sigma-Aldrich; $2 \mu \mathrm{l}$ of $5 \mathrm{mM}$ in PBS) into rats 10 minutes before and 1 hour after KA, according to previous methods $(26,27)$. Rats were euthanized 4 or 24 hours later, and brains were processed for histopathology and Western blotting (see below).

For examination of the effects of Akt inhibition, rats received icv infusions of the PI3K inhibitor LY294002 (50 nmol; Cell Signaling Technology Inc., Beverly, Massachusetts, USA) or vehicle (DMSO), 10 minutes before and 1 hour after KA or vehicle injection, as previously described (9). Brains obtained 72 hours after seizure termination were processed for immunostaining, DNA fragmentation analysis, and Western blotting (see below).

Induction of brief seizures in vivo. Additional rats were subjected to brief seizures via a Woodbury and Davenport electroshock apparatus (28). Maximal electroshock seizures (MESs) were delivered via orbital (corneal) electrodes using stimuli of $25 \mathrm{~V}, 500 \mathrm{~mA}$, and 0.2 seconds' duration. This paradigm induces a short (10- to 30-second), tonic-clonic convulsion that has been shown to downregulate proapoptotic $\mathrm{Bcl}-2$ family proteins and confer protection against status epilepticus (29). Rats were subjected to $1,3,6$, or 9 MESs over a period of 1-3 days and euthanized 24 hours after the last MES for histology and Western blotting. Control rats were handled and placed in contact with the electrodes without current.

In vitro seizure model, chromatin immunoprecipitation assay, and Bim antisense studies. Seizurelike activity was produced in hippocampal neuronal cultures using the method of Furshpan and Potter (30), as recently described (31). Briefly, hippocampal neurons from postnatal day $1-3$ rat pups were maintained in medium containing the glutamate antagonist kynurenic acid (Sigma-Aldrich) and high magnesium (11 $\mathrm{mM} \mathrm{MgCl}_{2}$ ) for 2-3 weeks. Seizurelike activity was induced for 40 minutes by placement of cultures into medium without kynurenic acid and high magnesium.

Binding of FKHR to the bim promoter site was determined using a chromatin immunoprecipitation assay, as described previously with modifications (32). Hippocampal cultures were treated with paraformaldehyde (2\%, 2 minutes) to cross-link proteins to DNA and then lysed and sonicated in buffer containing a protease inhibitor cocktail (Roche Molecular Biochemicals, Indianapolis, Indiana, USA). Samples were cleared by centrifugation $(12,000 \mathrm{~g})$ and then incubated with an antibody against FKHR (Cell Signaling Technology Inc.) overnight at $4^{\circ} \mathrm{C}$. A phospho-FKHR antibody was used as a negative control (Cell Signaling Technology Inc.). Protein-chromatin complexes were precipitated with protein A/Gagarose beads (Santa Cruz Biotechnology Inc., Santa Cruz, California, USA), and the DNA cross-linked to FKHR was released by heating in $0.1 \mathrm{M} \mathrm{NaHCO}_{3}$ for 16 hours at $65^{\circ} \mathrm{C}$. DNA samples were purified using a PCR clean-up kit (QIAGEN Inc., Valencia, California, USA), and then DNA was subjected to 36 cycles of PCR with primers specific to the FKHR consensus on the bim promoter: sense 5'-TCTCTCAACTGTCCTTCC-3', antisense 5'-TGTTTACCCTAGTGACTTGG-3' (accession number AY369780) (22). Amplicons were run on nondenaturing $10 \%$ acrylamide gels, stained with ethidium bromide, and viewed under UV light.

Bim expression was manipulated in vitro using an antisense approach. Antisense oligonucleotides complementary to the bim cDNA sequence were identified and synthesized by Sigma Genosys (Sigma-Aldrich) (antisense 1, 5' -TGGCCAAGCAACCTTCTG-3'; antisense 2, 5'-GTGTGACAGAGAAGGTGG-3'; antisense 3, 5' -TAAGGCAGTCTCAGGAGG-3'; antisense 4, 5' -ATTGCAGCCTGCTGAGAG-3'). Oligonucleotides were phosphorothioate modified on the first and last three linkages to reduce degradation. Hippocampal or cortical neuronal cultures were incubated for 48 hours with a cocktail of antisense ( $1 \mu \mathrm{M}$ of each) or control scrambled sequence $\left(5^{\prime}\right.$ TCGGCTAGACACCATCGT-3'; $4 \mu \mathrm{M}$ ). Cultures were processed for Western blotting (see below), or subjected to seizures and injury assessment using a lactate dehydrogenase (LDH) assay (31).

Human hippocampal samples. This study was approved by the Legacy Health System Institutional Review Board, and informed consent was obtained from all patients. Ten patients were referred for surgical resection of the temporal lobe by an epileptologist following extensive evaluation including neurological assessment, videoEEG recording, and MRI neuroimaging. Each patient was determined to have medically intractable epilepsy with a history of recurring seizures. However, no patient had experienced status epilepticus during the year in which surgery was performed. Clinical data from these patients are shown in Table 1. All patients were taking anticonvulsant medication prior to surgery. Patients underwent left or right temporal lobe resection. The hippocampus was dissected and separated from any adjacent temporal cortex $(\sim 15$ minutes), frozen in liquid nitrogen, and stored at $-70^{\circ} \mathrm{C}$ until use. Hippocampi were first sectioned for histology, and then coronal slabs of approximately $1 \mathrm{~mm}$ thickness were prepared from the remaining sample for biochemical analysis. Human control tissue $(n=6)$ was obtained from the Brain and Tissue Bank for Developmental Disorders at the University of Maryland (Baltimore, Maryland, USA) (see Table 1 for details). These specimens were similar fresh-frozen, en bloc hippocampi from people who died of causes not related to known neurological disease. Specimens were processed individually as described below for experimental tissue, and used for Western blotting and co-immunoprecipitation analysis.

Western blotting. Western blotting was performed as previously described (9). For in vivo models, rats were euthanized 0, 4, 8, 24, or 72 hours after administration of anticonvulsant in seizure animals, or 4,24 , or 72 hours after anticonvulsant in nonseizure controls, and ipsilateral hippocampus and cortex were obtained. Individual or pooled brain samples ( $n=2-3$ per group) were homogenized and lysed in buffer containing the protease inhibitor cocktail. Lysates were cleared by centrifugation, protein concentration was determined using Bradford reagent spectrophotometrically at $\mathrm{A}^{595} \mathrm{~nm}$, and $50-\mu \mathrm{g}$ samples were boiled in gel-loading buffer and separated on $12 \%$ SDS-PAGE gels. Proteins were trans- 
Table 1

Clinical data for controls and epilepsy patients

\begin{tabular}{lcccccc} 
& $\boldsymbol{n}$ & Age (yr) & Gender ratio (M/F) & PMI (h) & Seizure type & Seizure frequency (estimated per mo) \\
Controls & 6 & $29 \pm 7$ & $5: 1$ & $8 \pm 1$ & NA & NA \\
Epilepsy & 10 & $37 \pm 5$ & $3: 7$ & NA & CP (10), GTCS (3) & $7 \pm 3$ \\
\hline
\end{tabular}

M, male; F, female; PMI, postmortem interval; CP, complex partial; GTCS, generalized tonic-clonic seizures.

ferred to PVDF membranes (Bio-Rad Laboratories, Hercules, California, USA) and then incubated with antibodies against the following: 14-3-3 $\beta$, $\alpha$-tubulin, Bad, Bcl-2, Bax, p27 Kip1, FKHRL-1 (all from Santa Cruz Biotechnology Inc.), Bcl-xl (BD Biosciences, Lexington, Kentucky, USA), Bid (R\&D Systems Inc., Minneapolis, Minnesota, USA), Bim (Stressgen Biotechnologies Corp., Victoria, British Columbia, Canada), dynein (Chemicon International Inc., Temecula, California, USA), FKHR, phospho-Akt ${ }^{473}$, phosphoJNK $^{183,185}$, phospho-FKHR ${ }^{256}$, phospho-FKHRL-1 ${ }^{32}$, and phospho-p44/42 MAPK 202/204 (all from Cell Signaling Technology Inc.). Membranes were then incubated with HRP-conjugated secondary antibodies (1:2,000 dilution), and the proteins were detected by chemiluminescence (NEN Life Science Products Inc., Boston, Massachusetts, USA) and then exposed to BioMax film (Kodak Scientific Imaging Systems, Rochester, New York, USA).

Immunoprecipitation. Co-immunoprecipitation was performed as previously described (9). Control or seizure rats were euthanized 4 hours after administration of diazepam. Individual hippocampal samples for human studies or pooled hippocampal samples $(n=2$ per group) for rats were Dounce homogenized and lysed in buffer containing 1\% Nonidet P40 and the protease inhibitor cocktail. Protein samples $(0.5 \mathrm{mg})$ were incubated with $2 \mu \mathrm{g}$ of the immunoprecipitating antibody (Bim or Bcl-w) overnight at $4^{\circ} \mathrm{C}$ and then incubated with $1 \%$ BSA-cleared protein A/G-agarose beads (Santa Cruz Biotechnology Inc.) for 1 hour at $4{ }^{\circ} \mathrm{C}$. The protein-bead complex was then washed and collected by centrifugation, and samples were boiled in loading buffer, run on 12\% SDS-PAGE gels, probed with the antibody of interest, and processed as described for Western blotting. Positive controls (50 $\mu \mathrm{g}$ whole-cell lysate) and negative controls (omitting the immunoprecipitation antibody) were included to confirm specificity of reactions.

\section{Figure 1}

Seizure-induced hippocampal injury is associated with Bim overexpression. (A) Representative photomicrographs showing histopathology at 24 hours, in which marked loss of hippocampal CA3 neurons and the appearance of TUNEL are observed (top panels), while the cortex is largely spared (bottom panels). Inset in top left panel: Schematic of the hippocampal area from which images were taken (box). Inset in top right panel: $\times 100$ magnification of a TUNEL-stained CA3 cell. Scale bar: $75 \mu \mathrm{m}$. (B) Representative Western blot ( $n=2$ per lane) showing increased hippocampal Bim $\mathrm{EL}_{\mathrm{L}}$ levels following seizures in the rat. Molecular weight markers $(\mathrm{kDa})$ are given on the left, and time is shown in hours after termination of seizures. Staurosporine-treated rat C6 glioma cell lysates express all three Bim isoforms and are included as a positive control (C6+). $\alpha$-Tubulin is shown below to confirm equivalency of protein loading. (C) Graph showing quantification of changes in Bim $\mathrm{EL}_{\mathrm{L}}$ expression (OD units) after seizures. ${ }^{\star} P<0.05,{ }^{* \star} P<0.01$ vs. control (con). Data are from four rats per group. (D) Representative Western blot ( $n=2$ per lane) from cortex, revealing that Bim levels do not change in this region following seizures. Immunoblots are representative of two or three independent experiments.

Immunohistochemistry and DNA fragmentation analysis. Coronal brain sections at the level of bregma $-3.2 \mathrm{~mm}$ (33) were preblocked in $5 \%$ goat serum and then incubated overnight at $4{ }^{\circ} \mathrm{C}$ with antiFKHR (1:100). Sections were then washed three times in PBS and incubated for 2 hours at room temperature in a 1:500 dilution of goat anti-rabbit Cy3 (Jackson ImmunoResearch Laboratories Inc., West Grove, Pennsylvania, USA). Sections were then washed and mounted in medium containing 4',6-diamidino-2-phenylindole (Vector Laboratories Inc., Burlingame, California, USA) to assess nuclear morphology. Analysis of cells exhibiting DNA fragmentation was performed using fluorescein-linked TUNEL (Roche Molecular Biochemicals) to label double-stranded DNA breaks as previously described (9). Immunolabeling and TUNEL were stud-

A
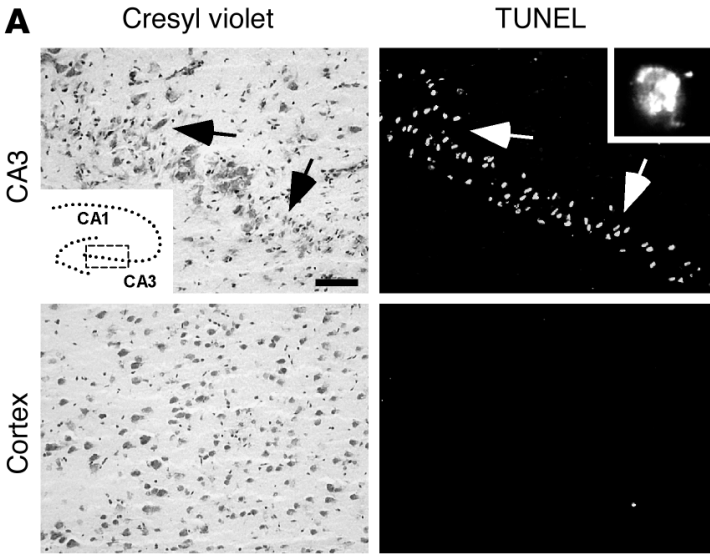

B

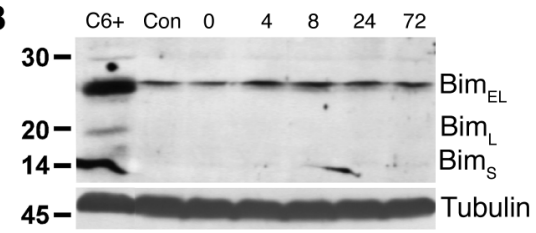

C

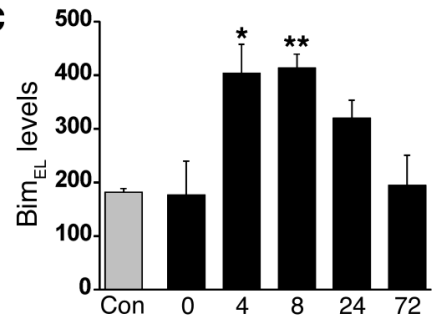

D

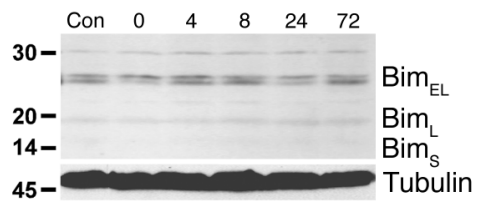



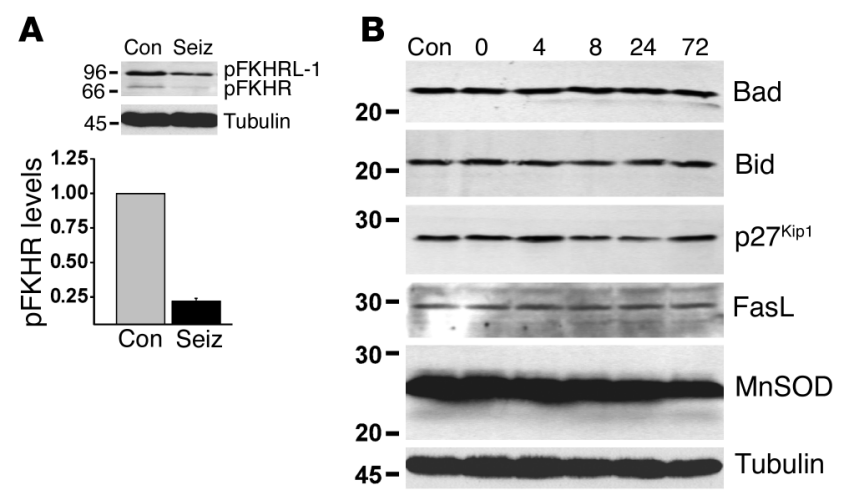

Figure 2

Activation of FKHR/FKHRL-1 following seizures. (A) Representative Western blot ( $n=2$ per lane) showing that levels of phosphorylated $(p)$ FKHRL-1 and FKHR are lower in hippocampal samples obtained 40 minutes after seizure induction (seiz) than in controls. The graph below shows quantitative change (fold vs. control) for pFKHR. ${ }^{*} P<0.05$ vs. control. Data are from six rats per group. (B) Representative Western blots ( $n=2$ per lane) showing that hippocampal expression levels of other BH3-only proteins (Bad and Bid) and forkhead targets (p27 Kip1, FasL, and manganese superoxide dismutase [MnSOD]) are not increased by seizures. Immunoblots are representative of two or three independent experiments.

ied using a Leica microscope (Wetzlar GmbH; Leica Microsystems Inc., Bannockburn, Illinois, USA) equipped for epifluorescent illumination under excitation/emission wavelengths of $340 / 425 \mathrm{~nm}$ (blue), 500/550 nm (green), and 580/630 nm (red).

Data analysis. Western blots were imaged using a Dage 72 camera (Dage-MTI Inc., Michigan City, Indiana, USA), and protein levels were determined using gel-scanning integrated optical density software (BIOQUANT Image Analysis Corp., Nashville, Tennessee, USA). Microscope images were captured using a DEI 750 threechip camera (Optronics, Goleta, California, USA) equipped with a BQ 8000 sVGA frame grabber (BIOQUANT Image Analysis Corp.) and analyzed using BIOQUANT. For the SOV experiment, neuron and TUNEL counts were performed for the entire cornu ammonis 3 (CA3), and a mean count was obtained from two adjacent sections. For the LY294002 study, five (for nuclear FKHR immunostaining) or ten (for TUNEL) $\times 40$ fields from the cortex were analyzed and a mean count obtained from adjacent sections. Similarly, TUNEL counts for MES studies (the sum of ten $\times 40$ fields) were performed on two adjacent sections and a mean count obtained. Data are presented as mean \pm SEM. Data were analyzed using the Mann-Whitney $U$ test or one-way ANOVA with post hoc Fisher's paired least significant difference test as appropriate (StatView software; SAS Institute Inc., Cary, North Carolina, USA). Significance was accepted at $P<0.05$.

\section{Results}

Seizure-induced neuronal death is associated with upregulation of Bim. Focally evoked seizures elicited by intra-amygdala KA caused the death of ipsilateral CA3 neurons and labeling by TUNEL 24 hours after seizure termination (Figure 1A). In contrast, the cortex exhibited very little cell death and TUNEL (Figure 1A).

We first performed Western blotting to determine the expression of Bim in control and seizure brain. At least three isoforms of Bim are generated as a result of alternative gene splicing, although the extra-long (EL) form is most common in neurons $(18,25)$. Western blot analysis revealed the presence of $\mathrm{Bim}_{\mathrm{EL}}$ in control rat hippocampus at approximately $26 \mathrm{kDa}$ (Figure 1B). The Bim long $\left(\mathrm{Bim}_{\mathrm{L}}\right)$ and short $\left(\mathrm{Bim}_{\mathrm{S}}\right)$ forms were expressed by rat $\mathrm{C} 6$ glioma cells (used as a positive control) but not in rat brain (Figure 1B). Seizures induced a significant increase in levels of $\mathrm{Bim}_{\mathrm{EL}}$ in rat brain from 4-8 hours after seizure cessation (Figure 1, B and C), while levels had returned to base line by 72 hours later. Bim expression in cortex was not significantly affected by seizures (Figure 1D).

Forkhead activation by seizures. Next, we sought to identify transcription factors that might be responsible for the upregulation of Bim after seizures. Although the transcription factor c-Jun can regulate Bim expression (18), its involvement here could be excluded because we previously demonstrated that c-Jun is not activated within the first 24 hours following seizures in our model (11). Accordingly, we focused on the involvement of the forkhead transcription factors that are also known to regulate Bim levels $(22,34$, 35 ). Western blotting revealed that FKHR and FKHRL-1 were constitutively expressed in rat brain and phosphorylated (Figure 2A). Analysis of hippocampal lysates obtained 40 minutes after seizure induction revealed that levels of both phospho-FKHR and phospho-FKHRL-1 were lower than in controls (Figure 2A). Levels of FKHR/FKHRL-1 phosphorylation at later survival times revealed some degree of recovery, although this was quite variable between animals (data not shown).

Next, we examined whether changes in Bim were specific to this particular BH3-only protein. Western blotting determined no expressional increases for either Bad or Bid in the hippocampus following seizures (Figure 2B).

To determine whether other genes under the transcriptional control of FKHR/FKHRL-1 are regulated by seizures, we examined expression of p $^{\text {Kip } 1}$, a cyclin-dependent kinase inhibitor, FasL, and manganese superoxide dismutase (MnSOD) $(23,35,36)$. Seizures caused a small increase in hippocampal p27Kip1 levels to

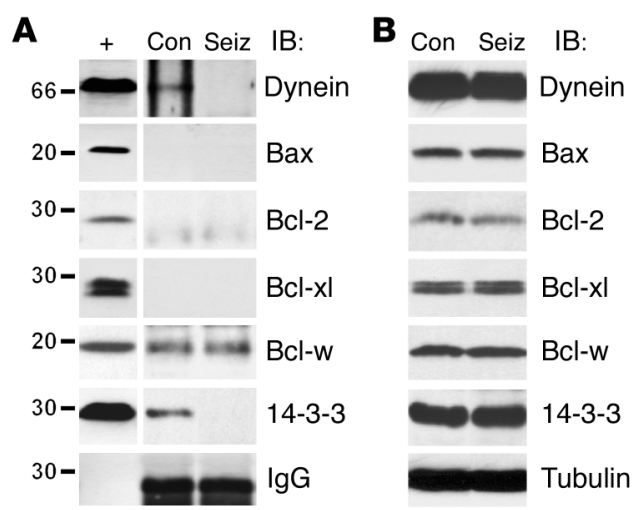

\section{Figure 3}

Bim interactions in control and seizure brain. (A) Bim was immunoprecipitated (IP) from control or seizure hippocampus 4 hours after diazepam administration ( $n=2$ per lane), and then lysates were immunoblotted (IB) to detect the presence of interacting proteins. Bim binds to dynein, Bcl-w, and 14-3-3 in control brain, but not Bax, Bcl-2, or Bcl-xl. Following seizures, Bim interactions with dynein and 14-3-3, but not Bcl-w, declined. IgG levels confirm equivalency of antibody loading. Whole cell lysates run concurrently are shown to the left to confirm molecular weight $(+)$. (B) Representative Western blots ( $n=2$ per lane) showing expression of each interacting protein in hippocampal lysates. Immunoblots are representative of two or three independent experiments. 
A
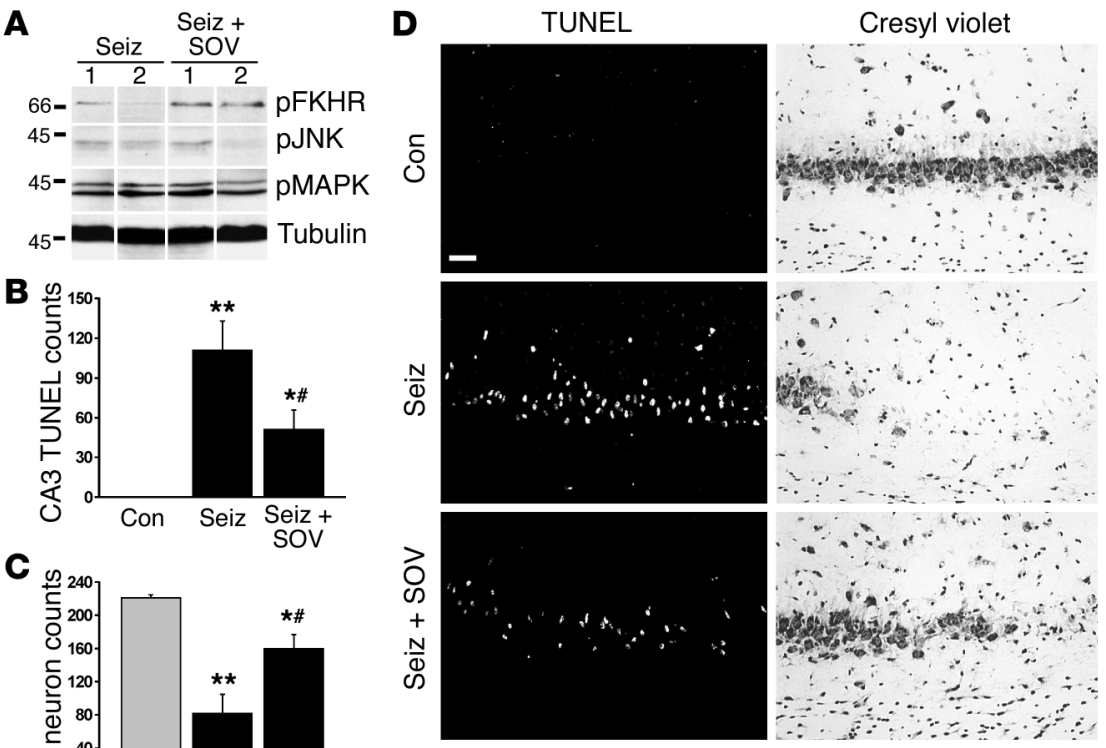

\section{Figure 4}

Neuroprotective effects of SOV. (A) Representative Western blots ( $n=1$ per lane) showing that phosphorylation of FKHR is higher in seizure rats given SOV (seiz + SOV) than in seizure rats given vehicle (seiz). SOV did not affect phosphorylation of JNK or MAPK. Data are from four rats per group. (B) Graph showing that hippocampal CA3 TUNEL counts at 24 hours were significantly lower in seizure rats that received SOV than in vehicle-injected seizure controls. ${ }^{*} P<0.05$ for seiz + SOV vs. seiz, ${ }^{\star \star} P<0.01$ for seiz vs. con, $\# P>0.05$ for seiz + SOV vs. con. (C) Survivingneuron counts from cresyl violet-stained sections at 24 hours revealed that SOV increased neuronal survival in CA3 compared with that in vehicle-treated seizures rats. ${ }^{*} P<0.05$ for seiz + SOV vs. seiz, ${ }^{*} P<0.001$ for seiz vs. con, ${ }^{\#} P>0.05$ for seiz + SOV vs. con. (D) Photomicrographs showing representative CA3 fields from TUNEL- and cresyl violet-stained sections for each group. Scale bar: $50 \mu \mathrm{m}$.
$132 \% \pm 3 \%$ of control at 4 hours after seizures, but levels were reduced to $37 \% \pm 10 \%$ of control at 24 hours ( $P<0.05$; Figure $2 \mathrm{~B})$. Hippocampal expression of FasL and MnSOD were not increased by seizures (Figure 2B).

Bim interacts with Bcl-w. We next examined several possible interactions of Bim following seizures using co-immunoprecipitation assays (9). Bim normally resides sequestered to the dynein complex but is released during apoptosis, whereupon it mediates its effects by neutralizing antiapoptotic Bcl-2 family proteins $(15,20)$. Bim was immunoprecipitated from control or seizure rat hippocampi at 4 hours, and then lysates were immunoblotted to detect interacting proteins. In control hippocampus, we detected a modest association of dynein and Bim (Figure 3A). In contrast, the interaction of dynein with Bim was not detected in seizure hippocampus; this suggested release or activation of Bim (Figure 3A). Next, we sought to identify which $\mathrm{Bcl}-2$ family proteins are targets of Bim. Bim did not interact with proapoptotic Bax in control or seizure hippocampus (Figure 3A). However, control hippocampal Bim immunoprecipitates contained detectable levels of antiapoptotic Bcl-w, but not Bcl-2 or Bcl-xl (Figure $3 \mathrm{~A}$ ). Seizures did not noticeably affect levels of $\mathrm{Bcl}-\mathrm{w}$ binding to Bim (Figure 3A). Finally, we examined whether Bim, like the other $\mathrm{BH} 3$-only proteins $\mathrm{Bad}$ and $\mathrm{Bid}$, interacts with the molecular chaperone 14-3-3 in control or seizure brain $(9,37)$. Using an antibody against the $\beta$ isoform, we detected modest binding of 14-3-3 to Bim immunoprecipitates in control hippocampus (Figure 3A). In contrast, the interaction of 14-3-3 with Bim was lower or lost in seizure samples (Figure 3A). Representative Western blots showing the largely unchanging expression of each protein tested are shown in Figure 3B.

SOV improves neuronal survival following seizures. It has previously been demonstrated that blocking of forkhead dephosphorylation with the nonspecific phosphatase inhibitor SOV reduces hippocampal neuronal death following ischemia (27). To gain insight into whether FKHR/FKHRL-1 activation, and, by inference, Bim, contribute significantly to seizure-induced neuronal death in vivo, we used a similar paradigm to block FKHR/FKHRL-1 dephosphorylation during seizures.
Western blotting on hippocampal extracts from seizure rats at 4 hours confirmed that levels of phosphorylated FKHR were $2.6 \pm 0.5$-fold higher in SOV-treated than in vehicle-treated animals $(P<0.05$; Figure $4 \mathrm{~A})$. In contrast, phosphorylation of JNK, $\mathrm{p} 44 / 42 \mathrm{MAPK}$, and Akt was not different between seizure rats treated with or without SOV (Figure 4A and data not shown).

Histopathological analysis of DNA fragmentation in brain sections obtained at 24 hours confirmed that seizures caused significant TUNEL staining in the ipsilateral hippocampal CA3 subfield (Figure 4, B and D). Treatment of seizure rats with SOV significantly reduced numbers of TUNEL-positive CA3 cells (Figure 4, B and D). Counts of surviving neurons complemented these findings, revealing that SOV led to a significant sparing of neurons compared with those of vehicle-treated seizure controls (Figure 4, C and D).

Seizures in vitro increase FKHR binding to the bim promoter, and Bim antisense reduces neuronal injury. Next, we sought direct evidence for forkhead targeting of the bim gene, and for the significance of Bim in promoting neuronal death after seizures. Using a model of seizurelike activity in vitro that is associated with programmed cell death (31), we established that seizures in this model also activate FKHR/FKHRL-1 and increase Bim expression (Figure 5A). Next, we investigated endogenous and seizure-affected binding of FKHR to the consensus promoter sequence on the bim gene using a modified chromatin immunoprecipitation assay (32). Chromatin fragments were immunoprecipitated with anti-FKHR or anti-phospho-FKHR and were analyzed by PCR. Under basal conditions, FKHR, but not phospho-FKHR, was associated with the bim forkhead promoter region (Figure 5B). One hour after seizures, we detected a consistent increase in FKHR association with the bim promoter region (Figure 5B).

Finally, we examined the effect of manipulation of Bim expression, using antisense oligonucleotides, on seizure-induced neuronal injury in vitro. Western blotting confirmed that 48 hours of pretreatment of hippocampal cultures with antisense oligonucleotides reduced Bim expression to about $25 \%$ of control (scrambled sequence-treated) levels, without altering levels of Bad or Bid (Figure 5C and data 

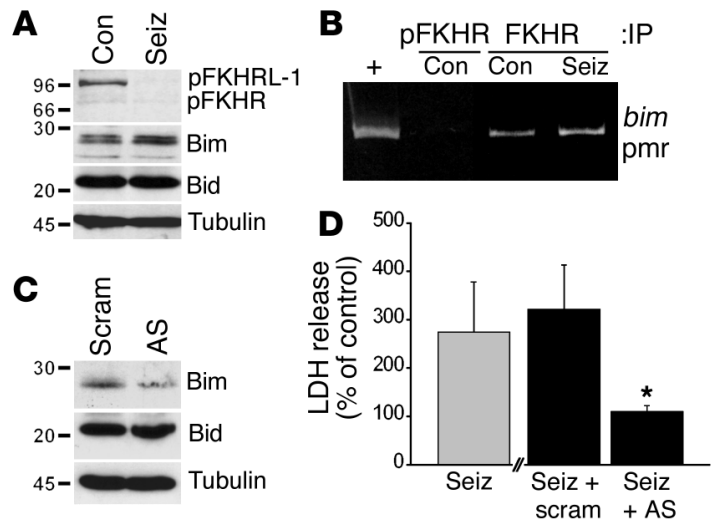

\section{Figure 5}

Seizures in vitro increase binding of FKHR to the bim promoter, and Bim antisense is neuroprotective. (A) Representative Western blots from hippocampal neuronal cultures at 24 hours, showing that seizurelike activity in vitro causes FKHR/FKHRL-1 dephosphorylation and increased Bim expression. Immunoblots showing unaffected Bid and $\alpha$-tubulin levels are included as controls. Immunoblots are representative of two or three independent experiments. (B) Chromatin immunoprecipitation assay demonstrating immunoprecipitation of the bim promoter (pmr) by FKHR. Chromatin fragments were immunoprecipitated using either anti-FKHR or anti-phospho-FKHR followed by PCR with primers specific for the bim-FKHR promoter region. Genomic DNA was subject to PCR as a positive (+) control. Phospho-FKHR did not precipitate the bim promoter. In contrast, FKHR precipitated the bim promoter, and this was increased in cultures subject to seizures and 1 hour of recovery. The image is representative of two independent experiments. (C) Representative Western blots confirming that Bim antisense (AS) reduces Bim levels compared with those in control cultures treated with a scrambled (scram) sequence. Immunoblots showing unaffected Bid and $\alpha$-tubulin levels are included as controls. Immunoblots are representative of two independent experiments. (D) Graph showing effects of Bim antisense on seizure-induced LDH release. Injury assessment at 24 hours revealed that seizures (seiz) elevated LDH levels to about $275 \%$ of control (base-line) levels. Incubation of cultures with the scrambled Bim antisense did not alter seizure-induced LDH release. In contrast, Bim antisense significantly reduced $L D H$ release following seizures. ${ }^{*} P<0.05$ for seiz + AS vs. seiz + scram.

not shown). Similar results were obtained when cortical neuronal cultures were used (data not shown). LDH release assays revealed that Bim antisense, but not the scrambled sequence, significantly reduced neuronal injury following seizurelike activity in vitro (Figure 5D).

Akt-mediated cortical neuroprotection is associated with inhibition of FKHR/Bim. Although Akt activation contributes to neuronal survival in the cortex following seizures (9), the mechanism of this protection is unknown. Since Akt can promote cell survival by inhibiting the activity of forkhead transcription factors (23), we tested whether Akt inhibits forkhead/Bim in the cortex following seizures. Akt activity was blocked in vivo using icv infusion of the PI3K inhibitor LY294002, and the cortex was studied 72 hours later as previously described (9). Using an antibody against FKHR, we first examined nuclear FKHR immunostaining in the cortex. Seizure animals that received vehicle did not exhibit cortical FKHR counts different from those of nonseizure controls, which confirmed that the cortex does not normally undergo FKHR activation after seizures (Figure 6, A and B). In contrast, cortical FKHR counts were significantly increased in seizure rats that received LY294002 (Figure 6, A and B). Next, we performed Western blotting to determine whether Bim levels were altered by LY294002. Immunoblotting revealed that Bim expression in LY294002-treated seizure rats was elevated to $119 \% \pm 4 \%$ of levels in seizure animals given vehicle $(P<0.05$; Figure $6 \mathrm{C})$. Finally, analysis of DNA fragmentation in the cortex determined that LY294002 significantly increased TUNEL counts compared with those in vehicle-treated seizure rats (Figure 6, D and E).

Akt activation, FKHR inbibition, and reduced Bim expression in buman temporal lobe epilepsy. We next examined whether the FKHR/Bim pathway is altered in hippocampi obtained after surgical resection for intractable seizures in epilepsy patients. Hippocampi from ten patients were compared with similarly processed hippocampi from six age-matched autopsy controls (see Table 1). Preliminary histopathological analysis of sections taken from epilepsy-patient samples confirmed a typical pattern of neuronal loss in the CA1, CA3, and hilus of the dentate gyrus (data not shown). TUNEL counts averaged about two per section, but numbers were not significantly different from those in controls (data not shown).

Western blot analysis revealed that hippocampal phospho-Akt levels were significantly higher in epilepsy samples compared with controls (Figure 7, A and B). Phospho-FKHR levels were significantly higher in epileptic brains than in controls, suggesting activation of Akt (Figure 7, A and C). The lack of phosphorylation in control samples was not an artifact of the tissue procurement process, since phospho-MAPK was detected in control specimens (data not shown).

Next, we examined expression of Bim and other BH3-only proteins. We detected constitutive expression of Bim in control hippocampal samples (Figure 7, D and E). Bim levels were significantly lower in epilepsy-patient specimens (Figure 7, D and E). Bad and Bid were also expressed in control hippocampi, but levels were not significantly different in epilepsy samples (Figure 7, D and E).

Binding of Bim to Bcl-w in human epileptic brain. To determine whether $\mathrm{Bcl}-\mathrm{w}$ is also a target of Bim in human brain, we immunoprecipitated Bcl-w from a subset of control and epilepsy-patient hippocampi and then performed Western blotting to detect associated Bim. Bcl$\mathrm{w}$ immunoprecipitates from control samples contained almost no Bim (Figure 8A). In contrast, we detected significant binding of Bim to Bcl-w in epilepsy samples (Figure 8, A and B). This appeared at a molecular weight of approximately $80 \mathrm{kDa}$, suggesting that this $\mathrm{Bcl}$ w-Bim complex exists as an oligomer. Western blotting confirmed that Bim and $\mathrm{Bcl}-\mathrm{w}$ were similarly expressed in the control and epilepsy samples used for this co-immunoprecipitation assay (Figure 8A).

As a result of these findings in human material, we immunoprecipitated Bcl-w from rat hippocampus and probed it for the presence of Bim. We found that in seizure rats, but not controls, Bim also bound Bcl-w at approximately $80 \mathrm{kDa}$ (Figure 8C).

Brief, noninjurious seizures mimic the molecular profile of the Bim pathway seen in epileptic brain. The Bim/FKHR expression profile in hippocampi from epilepsy patients suggests an adaptive response to frequent seizures. Since patients experience multiple short individual seizures rather than prolonged single seizure periods such as those induced by our in vivo KA model, we sought to model the human profile experimentally by evoking multiple brief seizures in rats. This was achieved with a Woodbury and Davenport electroshock apparatus. Preliminary tests (data not shown) and previous work using similar paradigms have proven that MESs are noninjurious and in fact protect the brain against damage induced by status epilepticus $(29,38,39)$.

TUNEL staining of brains obtained 24 hours after the last seizure determined that $1,3,6$, or 9 MESs did not cause significant DNA 
A

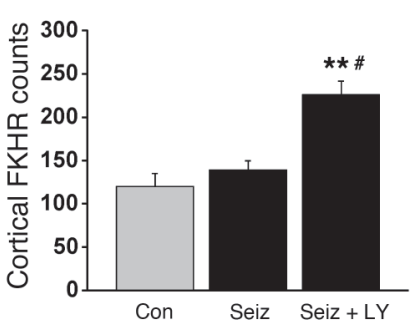

C

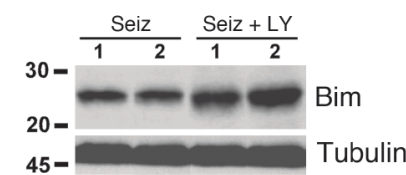

D

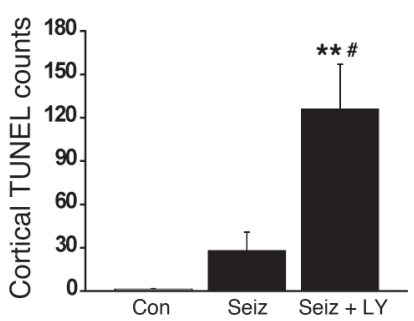

B

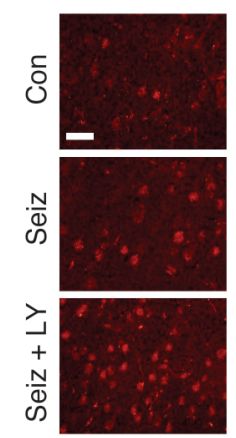

E

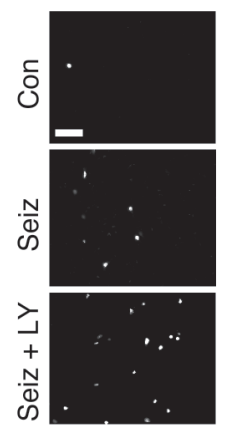

fragmentation in rat brain (count range $0-1$ per section for each group). Western blotting revealed that levels of phospho-FKHR, but not of phospho-Akt, were significantly correlated to the number of MESs (Figure 9A and data not shown). Western blotting also revealed that Bim expression was significantly inversely correlated to the number of MESs (Figure 9B). The specificity of these changes was confirmed by the fact that there was no correlation between the number of MESs and expression of the BH3-only proteins $\operatorname{Bad}(r=0.06, P=0.83)$ and $\operatorname{Bid}(r=0.24, P=0.42)$ (Figure 9C).

\section{Discussion}

The major finding of the present study is that programmed cell death pathways are differentially regulated after experimental seizures and in patients with temporal lobe epilepsy. Hippocampal injury following prolonged seizures is associated with FKHR/FKHRL-1 activation and increased Bim levels, while Bim is selectively downregulated in patients with temporal lobe epilepsy. These data suggest a molecular mechanism by which the epileptic brain may reduce its vulnerability to further neuronal loss. Indeed, priming brains by evoking multiple brief, noninjurious seizures reproduced this pattern. Bim regulation may therefore be a key determinant of neuronal survival, which suggests that its targeting might prevent neuronal loss after prolonged seizures and maintain epileptic brain in a state less susceptible to further cell loss.

\section{Figure 7}

Activation of Akt and inhibition of the Bim pathway in hippocampi from patients with temporal lobe epilepsy. (A) Representative Western blots showing higher hippocampal expression of phosphorylated Akt and FKHR in three epilepsy samples (epil) compared with three controls (con). (B and C) Graphs below show expression (OD units) of phosphorylated Akt (B) and FKHR (C) in control $(n=6)$ and epilepsy $(n=10)$ samples. (D) Representative Western blots showing that hippocampal expression of Bim is lower in epilepsy patient samples than in controls. In contrast, Bad and Bid are not differentially expressed. (E) Graph showing quantification of lower Bim expression in epilepsy samples. ${ }^{\star \star} P<0.01,{ }^{\star \star \star} P<0.0001$ vs. con.

\section{Figure 6}

Blocking Akt enables forkhead activation, Bim overexpression, and TUNEL in the cortex. (A) Graph showing nuclear FKHR counts in the cortex 72 hours after seizure termination. Seizure rats treated with vehicle did not exhibit different numbers of FKHR-positive cells in cortex compared with nonseizure controls. However, seizure rats that received LY294002 (seiz + LY) had significantly higher cortical FKHR counts. ${ }^{\star *} P<0.001$ vs. con, ${ }^{\#} P<0.01$ vs. seiz. Data are from five rats per group. (B) Representative cortical FKHR immunostaining (labeled with Cy3) from each group. Scale bar: $45 \mu \mathrm{m}$. (C) Representative Western blots ( $n=1$ or 2 per lane) showing cortical Bim expression in vehicle-treated (seiz) or LY294002-treated (seiz + LY) seizure rats. (D) Graph showing quantification of cortical TUNEL for each treatment group. ${ }^{* \star} P<0.001$ vs. con, ${ }^{\#} P<0.01$ vs. seiz. (E) Representative cortical TUNEL staining from each group. Scale bar: $50 \mu \mathrm{m}$.

The utility of neuroprotection to prevent brain damage in the wake of status epilepticus is generally recognized (40). Whether such adjuncts to anticonvulsant medication are warranted for epilepsy patients remains a source of debate, since it has been unclear whether neuronal loss occurs following brief seizures, affects the emergence of spontaneous seizures, or affects the progression of epilepsy $(2,41$, 42). Insight into several of these issues from experimental modeling suggests that neuroprotection may indeed benefit such patients. Studies have shown that brief seizures cause neurodegeneration (1), and, while only small numbers of neurons are lost, Sayin et al. pinpointed the onset of spontaneous seizures (epilepsy) following repetitive brief seizures to the time at which significant neuronal loss presented (6). Furthermore, neuronal loss is associated with the appearance of cognitive dysfunction following seizures (3) and may contribute to seizure severity in experimental epilepsy (5).

In pursuing how neurons die following seizures, we and others have shown that several caspases and auxiliary effectors of programmed cell death are activated, and that molecular and pharmacological manipulations of these are neuroprotective $(8,10,11,13)$ and may be able to modify epileptogenesis $(12,13)$. Although Bcl-2
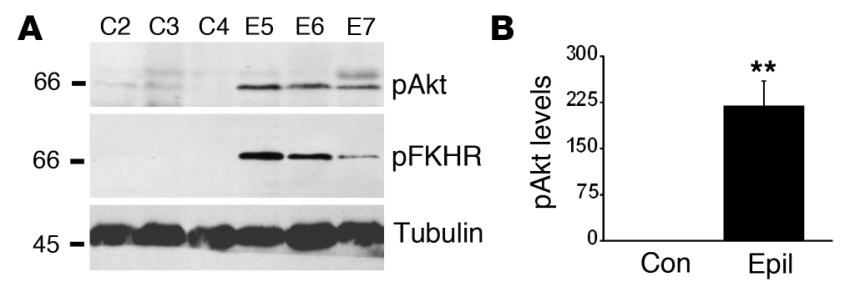

C

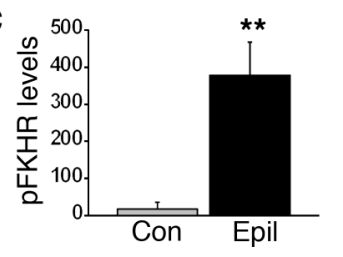

$\mathbf{E}$

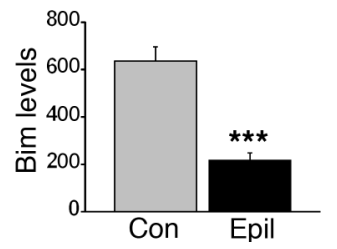

D

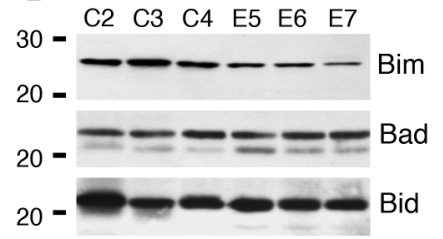


A

B
O
.$\frac{\overline{0}}{0}$
.$\frac{1}{0}$
$\frac{1}{0}$
$\frac{1}{3}$
$\frac{1}{0}$
0
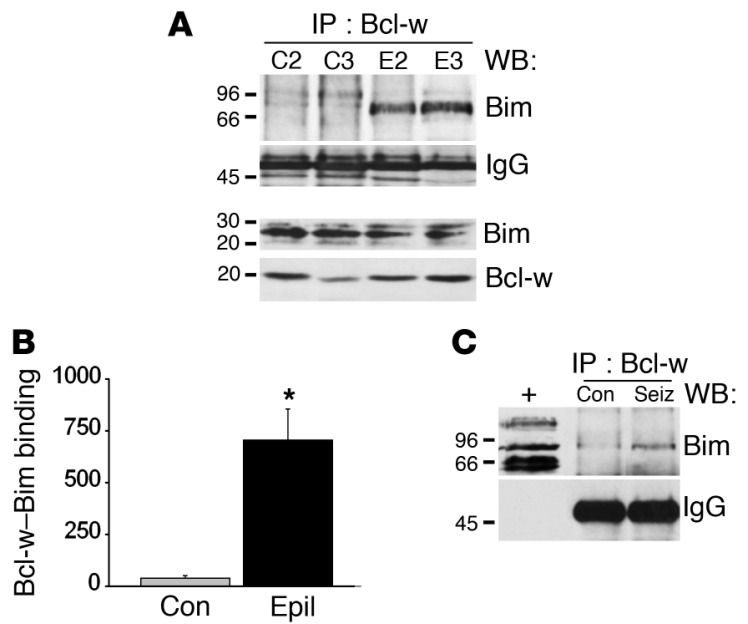

Figure 8

Binding of Bim to Bcl-w in hippocampi from patients with temporal lobe epilepsy. (A) Representative Western blot (WB) showing Bim binding to Bcl-w immunoprecipitates in epilepsy but not control brain samples. The IgG band confirms equivalency of antibody loading, and the immunoblots of Bim and Bcl-w below show total protein levels in these samples. (B) Graph showing quantification of higher Bim binding to Bcl-w (OD units) in epileptic brain. ${ }^{*} P<0.05$ vs. con. Data are from three patients per group. (C) Representative Western blot ( $n=2$ per lane) in which Bcl-w immunoprecipitates from rat seizure hippocampus at 4 hours are also shown to contain Bim as a high-weight complex. Whole cell lysates run concurrently are shown to the left to confirm molecular weight $(+)$. Immunoblots are representative of two independent experiments.

family proteins are key regulators of such pathways, their involvement in epileptic brain injury remains little understood, because seizure-induced expressional changes of $\mathrm{Bcl}-2$ family proteins often either are minimal or emerge beyond a time at which cell death could still be influenced (7). Bim has received increasing attention in the setting of neurological disease, since its overexpression is particularly common during neuronal death $(17,18,25)$. Our first experiments here showed that neuronal death after seizures is also associated with an early, transient increase in Bim expression. This was specific to this $\mathrm{BH} 3$-only protein, not a general response of forkhead-regulated genes, and appeared only in the damaged hippocampus. That such changes in Bim expression may contribute to neuronal death after seizures is supported by the potent neuroprotective effect of Bim knockdown in our in vitro antisense studies. Thus, our data support a selective involvement of the Bim pathway in the mediation of neuronal death following seizures, corroborating other findings that Bim promotes neuronal apoptosis (17).

Our binding assays support the prevailing view that Bim interacts with specific antiapoptotic Bcl-2 proteins $(15,16)$, although, like Bad (9), Bim is associated with 14-3-3 in normal hippocampus and is released during cell death. Bim did not bind proapoptotic Bax, and, while Bim can bind antiapoptotic Bcl-2 and Bcl-xl $(15,16)$, this did not occur before or after seizures. Rather, in both experimental and human material, Bim interacted with antiapoptotic Bcl-w. Increased binding of Bim to Bcl-w has recently been reported during apoptosis in conjunction with the formation of larger-molecular weight Bcl-w complexes (43). This is exactly what we observed in our human material, with similar findings in our experimental model. These human data suggest that Bim is activated in hip- pocampi from epilepsy patients, extending evidence for programmed cell death in temporal lobe resections from patients with intractable seizures (44). The cause of the specific matching of Bim and Bcl-w is unclear but may reside in their subcellular distributions, which are yet to be compared in this setting. These findings support the concept that Bcl-2 family proteins have specific "partners" in the brain and that Bim function might as effectively be manipulated by alteration of Bcl-w. Our results also reveal subtle distinctions from the prominent $\mathrm{Bcl}-2$ and $\mathrm{Bcl}-\mathrm{xl}$ upregulation that occurs following traumatic or ischemic brain injury (7), suggesting that therapeutic interventions might be tailored to specific insults.

The forkhead transcription factors regulate expression of several genes known to promote apoptosis (21), although antiapoptotic functions have been reported (45). Our findings extend work showing that brain injury activates FKHR/FKHRL-1 $(27,46)$. The immediacy of FKHR/FKHRL-1 activation after seizures suggests that it could account for increased Bim levels and cell death. Indeed, other transcription factors capable of increasing Bim expression are not activated in our model (11). Furthermore, FKHR/FKHRL-1 inhibition, while achieved using a nonspecific phosphatase inhibitor likely to affect other signaling pathways, was neuroprotective against seizure damage. Although the source of FKHR/FKHRL-1 activation after seizures is unknown, relevant stimuli could include PTEN (phosphatase and tensin homologue deleted on chromosome ten) (47) and ceramide (48), which are already implicated in seizure-induced neuronal death $(49,50)$.
A
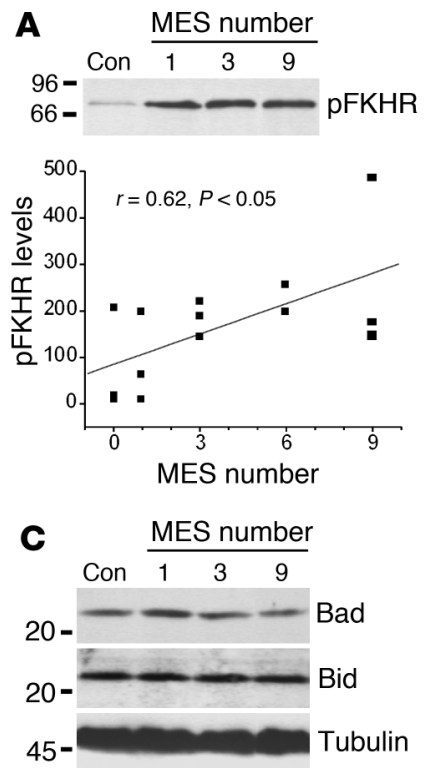
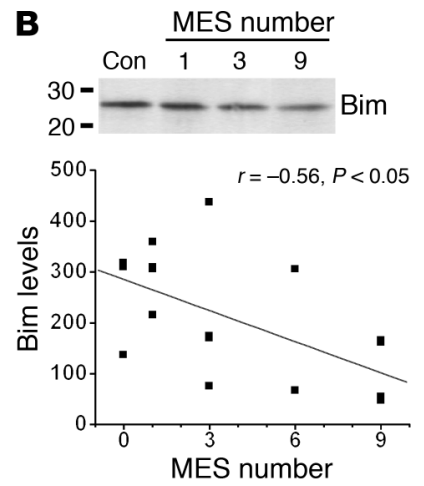

\section{Figure 9}

Brief electroshock seizures model the Bim/FKHR response seen in epilepsy patients. (A) Representative Western blot $(n=1$ or 2 per lane) showing increased pFKHR levels in rat brain in proportion to MES number. The graph below shows linear regression analysis of pFKHR levels (OD units) in relation to MES number. Data are from three or four rats per group. (B) Representative Western blot $(n=1$ or 2 per lane) showing reduction in Bim levels in rat brain in proportion to MES number. Linear regression analysis in the graph below shows the decline of Bim expression in relation to MES number. (C) Representative Western blots ( $n=1$ or 2 per lane) showing that levels of Bad and Bid are unaffected by MES. 
The function of FKHR/FKHRL-1 is opposed by Akt, which phosphorylates and inactivates FKHR/FKHRL-1 $(24,51)$. This appears to occur in the spared cortex following seizures, since LY294002, an inhibitor of the upstream PI3K, increased nuclear FKHR immunostaining, Bim expression, and TUNEL in this region. Accordingly, these data provide a mechanistic explanation for the protection of the cortex following seizures and extend evidence that Akt is a key survival factor following brain injury $(52,53)$. The trigger for Akt activation after seizures is unknown, but cytokines that are expressed in the brain can activate this pathway (54). Why hippocampal Akt remains quiescent is unclear (9), but forced Akt activation in this region might prevent seizure-induced neuronal loss.

Whether the brief seizures experienced by epilepsy patients are harmful to the brain remains a critical question in the treatment of this disease $(2,55)$. Our study provides the first demonstration that expression and activity of Bim, FKHR, and Akt is altered in the hippocampus of patients with intractable seizures. Surprisingly, the lowered Bim expression, inactivated FKHR, and active Akt in the epileptic brain formed a pattern opposite to that of the molecular response to prolonged (damaging) seizures in the rat. If elevations in Bim are responsible for neuronal death after prolonged seizures, these clinical data could reflect a mechanism by which the epileptic brain selectively adjusts its repertoire of proapoptotic proteins to reduce vulnerability to future events. Indeed, TUNEL staining was limited in our temporal lobe resections in this study, and previously (44). This hypothesis is supported by our demonstration that noninjurious electroshock seizures, which are able to protect the brain from prolonged seizures (29), replicated the profile of Bim and FKHR changes seen in the human epileptic brain. Unfortunately, clinical neuroimaging evidence for continuing hippocampal damage in such patients (55), combined with the observed apoptosis-associated Bim-Bcl-w complex, suggests that these countermeasures to diminish vulnerability are at best only partly effective. Accordingly, neuroprotective interventions could provide the necessary assistance to the brain's endogenous capacity to react to the harmful environment presented by repetitive seizures.

Some limitations should be considered in the interpretation of our findings. It remains possible that other $\mathrm{Bcl}-2$ family proteins that did not appear to bind Bim in co-immunoprecipitation assays could nevertheless form oligomers following seizures. Second, our epilepsy and control human material were suitably matched in terms of gender and age but not for the effects of autopsy delay or influences of antiepileptic drugs, surgery, and anesthesia. However, internal controls (unaltered Bad and Bid expression) make erroneous conclusions arising from such factors unlikely; and control rats in our experimental model undergo surgical manipulation for placement of cannulas and electrodes and receive anticonvulsant, and this does not modulate cell death pathways $(8,9,44)$.

With the therapeutic use of brain stimulation for the treatment of epilepsy (56), our MES findings suggest that such interventions may alter brain levels of Bim, FKHR, and/or Akt. Whether this may have any benefit (or indeed detriment) for patients is unknown. Finally, Bak, which has a $\mathrm{BH} 3$ and a transmembrane domain in common with Bim, has been shown to have direct effects on neuronal excitability (57). If Bim possesses similar properties, its downregulation may be affecting the excitability of the epileptic brain.

In conclusion, our study reveals that Bim may be a molecular rheostat of injury susceptibility that contributes to neuronal loss after prolonged seizures while being downregulated as a means to influence vulnerability in the brain subject to frequent brief seizures. Accordingly, this pathway might be targeted to treat brain injuries known to precipitate epilepsy, or to form neuroprotective adjuncts to anticonvulsants for epilepsy patients who suffer from frequent seizures.

\section{Acknowledgments}

The authors would like to thank R. Rosenbaum and K. Abtin for surgical collection of specimens, and Stephani B. Williams, Dan J. Torrey, and Ron Geison (Sigma-Aldrich) for technical support. This work was supported by NIH/National Institute of Neurological Disorders and Stroke grants NS39016 (to D.C. Henshall and R.P. Simon) and NS41935 (to D.C. Henshall).

Received for publication September 5, 2003, and accepted in revised form January 13, 2004.

Address correspondence to: David C. Henshall, Robert S. Dow Neurobiology Laboratories, Legacy Clinical Research \& Technology Center, 1225 NE Second Avenue, Portland, Oregon 97232, USA. Phone: (503) 413-5260; Fax: (503) 413-5465; E-mail: dhenshall@DowNeurobiology.org.
1. Bengzon, J., Mohapel, P., Ekdahl, C.T., and Lindvall, O. 2002. Neuronal apoptosis after brief and prolonged seizures. Prog. Brain Res. 135:111-119.

2. Pitkanen, A., and Sutula, T.P. 2002. Is epilepsy a progressive disorder? Prospects for new therapeutic approaches in temporal-lobe epilepsy. Lancet Neurol. 1:173-181.

3. Kotloski, R., Lynch, M., Lauersdorf, S., and Sutula, T. 2002. Repeated brief seizures induce progressive hippocampal neuron loss and memory deficits. Prog. Brain Res. 135:95-110.

4. Jokeit, H., et al. 1999. Bilateral reductions of hippocampal volume, glucose metabolism, and wada hemispheric memory performance are related to the duration of mesial temporal lobe epilepsy. J. Neurol. 246:926-933.

5. Zhang, X., et al. 2002. Relations between brain pathology and temporal lobe epilepsy. J. Neurosci. 22:6052-6061.

6. Sayin, U., Osting, S., Hagen, J., Rutecki, P., and Sutula, T. 2003. Spontaneous seizures and loss of axo-axonic and axo-somatic inhibition induced by repeated brief seizures in kindled rats. J. Neurosci. 23:2759-2768.
7. Liou, A.K.F., Clark, R.S., Henshall, D.C., Yin, X.M., and Chen, J. 2003. To die or not to die for neurons in ischemia, traumatic brain injury and epilepsy: a review on the stress-activated signaling pathways and apoptotic pathways. Prog. Neurobiol. 69:103-142.

8. Henshall, D.C., et al. 2001. Cleavage of Bid may amplify caspase-8-induced neuronal death following focally evoked limbic seizures. Neurobiol. Dis. 8:568-580.

9. Henshall, D.C., et al. 2002. Activation of Bcl-2-associated death protein and counter-response of Akt within cell populations during seizure-induced neuronal death. J. Neurosci. 22:8458-8465.

10. Roy, M., Hom, J.J., and Sapolsky, R.M. 2002. HSVmediated delivery of virally derived anti-apoptotic genes protects the rat hippocampus from damage following excitotoxicity, but not metabolic disruption. Gene Ther. 9:214-219.

11. Shinoda, S., et al. 2003. Formation of a tumour necrosis factor receptor 1 molecular scaffolding complex and activation of apoptosis signal-regulating kinase 1 during seizure-induced neuronal death. Eur. J. Neurosci. 17:2065-2076.

12. Moriwaki, A., Lu, Y.F., Tomizawa, K., and Matsui,
H. 1998. An immunosuppressant, FK506, protects against neuronal dysfunction and death but has no effect on electrographic and behavioral activities induced by systemic kainate. Neuroscience. 86:855-865.

13. Narkilahti, S., Nissinen, J., and Pitkanen, A. 2003. Administration of caspase 3 inhibitor during and after status epilepticus in rat: effect on neuronal damage and epileptogenesis. Neuropharmacology. 44:1068-1088.

14. Adams, J.M., and Cory, S. 1998. The Bcl-2 protein family: arbiters of cell survival. Science. 281:1322-1326.

15. O'Connor, L., et al. 1998. Bim: a novel member of the $\mathrm{Bcl}-2$ family that promotes apoptosis. $E M B O J$. 17:384-395.

16. Hsu, S.Y., Lin, P., and Hsueh, A.J. 1998. BOD (Bcl-2related ovarian death gene) is an ovarian $\mathrm{BH} 3$ domain-containing proapoptotic $\mathrm{Bcl}-2$ protein capable of dimerization with diverse antiapoptotic Bcl-2 members. Mol. Endocrinol. 12:1432-1440.

17. Putcha, G.V., et al. 2001. Induction of BIM, a proapoptotic BH3-only BCL-2 family member, is critical for neuronal apoptosis. Neuron. 29:615-628.

18. Whitfield, J., Neame, S.J., Paquet, L., Bernard, O., 
and Ham, J. 2001. Dominant-negative c-Jun promotes neuronal survival by reducing BIM expression and inhibiting mitochondrial cytochrome $c$ release. Neuron. 29:629-643.

19. Bouillet, P., Cory, S., Zhang, L.C., Strasser, A., and Adams, J.M. 2001. Degenerative disorders caused by Bcl-2 deficiency prevented by loss of its BH3-only antagonist Bim. Dev. Cell. 1:645-653.

20. Puthalakath, H., Huang, D.C., O’Reilly, L.A., King, S.M., and Strasser, A. 1999. The proapoptotic activity of the Bcl-2 family member Bim is regulated by interaction with the dynein motor complex. $\mathrm{Mol}$. Cell. 3:287-296.

21. Burgering, B.M., and Kops, G.J. 2002. Cell cycle and death control: long live Forkheads. Trends Biochem. Sci. 27:352-360.

22. Gilley, J., Coffer, P.J., and Ham, J. 2003. FOXO transcription factors directly activate bim gene expression and promote apoptosis in sympathetic neurons. J. Cell Biol. 162:613-622.

23. Brunet, A., et al. 1999. Akt promotes cell survival by phosphorylating and inhibiting a Forkhead transcription factor. Cell. 96:857-868.

24. Tang, E.D., Nunez, G., Barr, F.G., and Guan, K.-L. 1999. Negative regulation of the forkhead transcription factor FKHR by Akt. J. Biol. Chem. 274:16741-16746.

25. Shibata, M., et al. 2002. Temporal profiles of the subcellular localization of Bim, a BH3-only protein, during middle cerebral artery occlusion in mice. J. Cereb. Blood Flow Metab. 22:810-820.

26. Kawano, T., et al. 2001. Neuroprotective effect of sodium orthovanadate on delayed neuronal death after transient forebrain ischemia in gerbil hippocampus. J. Cereb. Blood Flow Metab. 21:1268-1280.

27. Kawano, T., et al. 2002. Decreased akt activity is associated with activation of forkhead transcription factor after transient forebrain ischemia in gerbil hippocampus. J. Cereb. Blood Flow Metab. 22:926-934.

28. Woodbury, L.A., and Davenport, V.D. 1952. Design and use of a new electroshock seizure apparatus, and analysis of factors altering seizure threshold and pattern. Arch. Int. Pharmacodyn. Ther. 92:97-104.

29. Kondratyev, A., Sahibzada, N., and Gale, K. 2001. Electroconvulsive shock exposure prevents neuronal apoptosis after kainic acid-evoked status epilepticus. Brain Res. Mol. Brain Res. 91:1-13.

30. Furshpan, E.J., and Potter, D.D. 1989. Seizure-like activity and cellular damage in rat hippocampal neurons in cell culture. Neuron. 3:199-207.
31. Meller, R., et al. 2003. Seizure-like activity leads to the release of BAD from 14-3-3 protein and cell death in hippocampal neurons in vitro. Cell Death Differ. 10:539-547.

32. Impey, S., et al. 2002. Phosphorylation of CBP mediates transcriptional activation by neural activity and CaM kinase IV. Neuron. 34:235-244.

33. Paxinos, P., and Watson, C. 1997. The rat brain in stereotaxic coordinates. Academic Press Inc. London, United Kingdom.

34. Dijkers, P.F., Medema, R.H., Lammers, J.W., Koenderman, L., and Coffer, P.J. 2000. Expression of the pro-apoptotic Bcl-2 family member Bim is regulated by the forkhead transcription factor FKHR-L1 Curr. Biol. 10:1201-1204.

35. Stahl, M., et al. 2002. The forkhead transcription factor FoxO regulates transcription of $\mathrm{p} 27 \mathrm{Kip} 1$ and Bim in response to IL-2. J. Immunol. 168:5024-5031.

36. Kops, G.J., et al. 2002. Forkhead transcription factor FOXO3a protects quiescent cells from oxidative stress. Nature. 419:316-321.

37. Shinoda, S., et al. 2003. Interaction of 14-3-3 with Bid during seizure-induced neuronal death. J. New rochem. 86:460-469.

38. Kelly, M.E., and McIntyre, D.C. 1994. Hippocampa kindling protects several structures from the neuronal damage resulting from kainic acid-induced status epilepticus. Brain Res. 634:245-256

39. Khan, A., Lai, H., Nishimura, Y., Mirolo, M.H., and Singh, N.P. 1994. Effects of ECS on DNA singlestrand breaks in rat brain cells. Convuls. Ther. 11:114-121.

40. Meldrum, B.S. 2002. Implications for neuroprotective treatments. Prog. Brain Res. 135:487-495.

41. Aminoff, M.J. 1998. Do nonconvulsive seizures damage the brain? No. Arch. Neurol. 55:119-120.

42. Sutula, T.P., and Pitkanen, A. 2001. More evidence for seizure-induced neuron loss: is hippocampal sclerosis both cause and effect of epilepsy? Neurology. 57:169-170

43. Wilson-Annan, J., et al. 2003. Proapoptotic BH3only proteins trigger membrane integration of prosurvival Bcl-w and neutralize its activity. J. Cell Biol. 162:877-888.

44. Henshall, D.C., et al. 2000. Alterations in bcl-2 and caspase gene family protein expression in human temporal lobe epilepsy. Neurology. 55:250-257.

45. Kops, G.J.P.L., et al. 2002. Forkhead transcription factor FOXO3a protects quiescent cells from oxidative stress. Nature. 419:316-321.
46. Jenkins, L.W., et al. 2002. Conventional and functional proteomics using large format two-dimensional gel electrophoresis 24 hours after controlled cortical impact in postnatal day 17 rats. J. Neurotrauma. 19:715-740.

47. Nakamura, N., et al. 2000. Forkhead transcription factors are critical effectors of cell death and cell cycle arrest downstream of PTEN. Mol. Cell. Biol. 20:8969-8982.

48. Stoica, B.A., Movsesyan, V.A., Lea, P.M., 4th, and Faden, A.I. 2003. Ceramide-induced neuronal apoptosis is associated with dephosphorylation of Akt, BAD, FKHR, GSK-3beta, and induction of the mitochondrial-dependent intrinsic caspase pathway. Mol. Cell. Neurosci. 22:365-382.

49. Gary, D.S., and Mattson, M.P. 2002. PTEN regulates Akt kinase activity in hippocampal neurons and increases their sensitivity to glutamate and apoptosis. Neuromolecular Med. 2:261-269.

50. Mikati, M.A., et al. 2003. Hippocampal programmed cell death after status epilepticus: evidence for NMDA-receptor and ceramide-mediated mechanisms. Epilepsia. 44:282-291.

51. Dijkers, P.F., et al. 2002. FKHR-L1 can act as a critical effector of cell death induced by cytokine withdrawal: protein kinase B-enhanced cell survival through maintenance of mitochondrial integrity. J. Cell Biol. 156:531-542.

52. Ouyang, Y.B., et al. 1999. Survival- and death-promoting events after transient cerebral ischemia: phosphorylation of Akt, release of cytochrome C and activation of caspase-like proteases. J. Cereb. Blood Flow Metab. 19:1126-1135.

53. Noshita, N., Lewen, A., Sugawara, T., and Chan, P.H. 2002. Akt phosphorylation and neuronal survival after traumatic brain injury in mice. Neurobiol. Dis. 9:294-304.

54. Kaplan, D.R., and Miller, F.D. 2000. Neurotrophin signal transduction in the nervous system. Curr. Opin. Neurobiol. 10:381-391.

55. Duncan, J.S. 2002. MRI studies. Do seizures damage the brain? Prog. Brain Res. 135:253-261.

56. Murphy, J.V., and Patil, A. 2003. Stimulation of the nervous system for the management of seizures: current and future developments. CNS Drugs. 17:101-115.

57. Fannjiang, Y., et al. 2003. BAK alters neuronal excitability and can switch from anti- to pro-death function during postnatal development. Dev. Cell. 4:575-585 